\title{
Pulmonary hypertension in systemic lupus erythematosus: a systematic review and analysis of 642 cases in Chinese population
}

\author{
Y. K. Xia $\cdot$ S. H. Tu $\cdot$ Y. H. Hu $\cdot$ Y. Wang $\cdot$ \\ Z. Chen $\cdot$ H. T. Day $\cdot$ K. Ross
}

Received: 13 March 2012/Accepted: 23 August 2012/Published online: 16 September 2012

(C) The Author(s) 2012. This article is published with open access at Springerlink.com

\begin{abstract}
Pulmonary hypertension (PH) is an increasingly recognized complication of systemic lupus erythematosus (SLE). To develop a more comprehensive understanding of the clinical and pathological characteristics of pulmonary hypertension associated with systemic lupus erythematosus (PH/SLE) in the Chinese population, a systematic review of the literature up to 2012 was conducted. Six hundred and forty-two Chinese PH/SLE cases from 22 studies were identified as well documented and further analyzed. Transthoracic echocardiography (TTE), X-ray, electrocardiogram and right heart catheterization (RHC) were performed to diagnose PH in SLE patients. The mean age of subjects was 35.5 years, the male to female ratio was $1: 14$, and the mean duration of SLE when PH was diagnosed was 10.7 years. The prevalence of PH in SLE was 2.8-23.3\%. Symptoms were usually nonspecific, and the observed clinical characteristics include Raynaud's phenomenon $(41.4 \%)$, serous effusion $(27.7 \%)$, positive RNP $(51.5 \%)$ and positive ACL $(46.6 \%)$. Gold standard RHC is strongly recommended, especially for those who had resting pulmonary arterial systolic pressure $>30 \mathrm{mmHg}$ on TTE with the aforementioned clinical characteristics. Corticosteroids,
\end{abstract}

Y. K. Xia · S. H. Tu ( $₫)$ · Y. H. Hu · Y. Wang · Z. Chen Department of Integrated Chinese and Western Medicine,

Tongji Hospital of Tongji Medical College, Central-China (Huazhong) University of Science and Technology, 1095 Jiefang Avenue, Wuhan 430030, Hubei,

People's Republic of China

e-mail: shtutjh@yahoo.cn; Shtu@tjh.tjmu.edu.cn

H. T. Day

ODS Health Plans, Portland, OR, USA

K. Ross

Oregon State University, Corvallis, OR, USA immunosuppressants and vasodilators were the most common medications employed in treatment. Early identification and standard PH treatment with intensive SLE treatment can improve the prognosis.

Keywords Pulmonary hypertension - Systemic lupus erythematosus - Raynaud's phenomenon .

Serous effusion - Right heart catheterization - Prognosis

\section{Introduction}

Pulmonary hypertension ( $\mathrm{PH})$ is a severe, potentially lifethreatening complication of systemic lupus erythematosus (SLE) $[1,2]$. The reported prevalence of PH in SLE ranged from 0.5 to $14 \%$ in a previous review of the literature [3]. The mechanisms involved in the pathogenesis of $\mathrm{PH}$ are largely unknown. Elevated antiendothelial cell antibodies (aECA) were detected and reported in patients with SLE, especially with $\mathrm{PH}$, which implied that aECA may be involved in the pathogenesis of vascular injury [4]. The association of $\mathrm{PH}$ with SLE suggests an underlying inflammatory component in the pathogenesis of this syndrome [5].

In SLE, the prognosis of patients with $\mathrm{PH}$ has been reported to be very poor and the mean survival from the onset of $\mathrm{PH}$ was 2 years [6-9]. PH is the third most common cause of death in SLE following infection and organ failure [10]. With the widespread availability of high-quality imaging systems and a better understanding of $\mathrm{PH}$ pathology [11, 12], the number of such patients reported in the Chinese literature has been steadily increasing. To better understand PH in SLE and to raise more awareness and help clinicians, a systematic review of the literature up to 2012 was conducted; clinical and pathological characteristics of PH associated with SLE 
were examined; well-documented Chinese cases were identified and further analyzed with the aim to provide a more comprehensive understanding of PH in SLE in the Chinese population.

\section{Methods and materials}

A comprehensive literature search was conducted to retrieve up-to-date $\mathrm{PH}$ in SLE-related information up to 2012 from multiple sources including the Chinese Biology and Medicine Database (CBMD), China Hospital Knowledge Database (CHKD), Wanfang Database, VIP Information Database, PubMed, Embase, Web of Science and Ovid. Indexing keywords used for the search and retrieval included PH, PAH, PHT, secondary pulmonary hypertension, primary pulmonary hypertension, lupus and systemic lupus erythematosus.

With a clear focus on PH in SLE in the Chinese population, all retrieved articles were carefully reviewed and checked for possible duplication to avoid the repetitive adoption of the same data and materials. The inclusion criteria were made that SLE diagnosis has to meet at least the 1982 revised ACR criteria of SLE [13] and diagnosis of $\mathrm{PH}$ was made when the resting pulmonary arterial systolic pressure (PASP) was $>30 \mathrm{mmHg}$ at rest by TTE (transthoracic echocardiography). Excluded were other forms of $\mathrm{PH}$ due to left heart disease, chronic lung disease and/or hypoxemia, chronic thromboembolism and a miscellaneous group as well as $\mathrm{PH}$ associated with other autoimmune diseases (such as systemic sclerosis, mixed connective tissue disease and so on).

All unduplicated cases meeting the above inclusion and exclusion criteria were entered into a database. Cases were considered well documented if they have at least 5 of the following 7 categories of information: age, gender, symptoms, imaging, laboratory tests, treatment data (from the clinical records) and follow-up. A total of 22 studies with 642 well-documented PH-SLE cases were included and analyzed. All statistical analyses were performed using SPSS 11.0 for Windows.

\section{Results}

Twenty-two studies with 642 well-documented PH-SLE cases were included in this analysis. Thirteen studies $(59.1 \%)$ were from Beijing, Shanghai and Guangzhou (the capital of Guangdong province, adjacent to Hong Kong). A sum of 430 patients came from these three cities, representing two-thirds $(67 \%)$ of the total 642 cases. Information about patients meeting the well-documented SLE-PH criteria is listed in Table 1.
Table 1 Patients meeting the well-documented SLE-PAH criteria

\begin{tabular}{llr}
\hline Criteria & $n$ & $\%$ \\
\hline Age (years) & 642 & 100.0 \\
Gender & 642 & 100.0 \\
Symptoms & 466 & 72.6 \\
Imaging & 642 & 100.0 \\
Laboratory tests & 532 & 82.9 \\
Treatment & 273 & 42.5 \\
Follow-up & 266 & 41.4 \\
\hline
\end{tabular}

Total $n=642$

Of the 642 patients, 42 were males and 600 were females. The male to female ratio was $1: 14$. Their age ranged from 8 to 85 years (with a mean age of 35.5), and their disease duration ranged from 0.5 to 50.5 years (with a mean of 10.7 years). The prevalence rate of PH in SLE reported in 12 studies ranged from 2.8 to $23.3 \%$.

The symptoms were usually nonspecific, and often coexisted with several clinical characteristics. The three most commonly observed were Raynaud's phenomenon, arthritis and serous effusion (Table 2).

One hundred and ninety-three patients (41.4\%) from 17 studies had Raynaud's phenomenon. In seven studies, $51.2 \%(32.4-77.8 \%)$ of lupus patients with $\mathrm{PH}$ had Raynaud's phenomenon versus $19.9 \%(10.3-36 \%)$ of

Table 2 Symptoms of PAH associated with SLE patients $(n=466)$

\begin{tabular}{lcc}
\hline Symptoms & Patients $(n)$ & $\%$ \\
\hline Raynaud's phenomenon & 193 & 41.4 \\
Arthritis & 178 & 38.2 \\
Serous effusion & 129 & 27.7 \\
Serositis & 101 & 21.7 \\
Pulmonary fibrosis & 83 & 17.8 \\
Polypnea & 58 & 12.4 \\
Fatigue & 57 & 12.2 \\
Rash & 49 & 10.5 \\
Acra vasculitis & 37 & 7.9 \\
Fever & 29 & 6.2 \\
Oral ulcer & 24 & 5.2 \\
Alopecie & 20 & 4.3 \\
Chest distress & 16 & 3.4 \\
P2 accentuation & 15 & 3.2 \\
Butterfly erythema & 12 & 2.6 \\
Photosensitivity & 11 & 2.4 \\
Palpitation & 10 & 2.1 \\
Dry mouth and eye & 9 & 1.9 \\
Limbs edema & 9 & 1.9 \\
Dyspnea & 6 & 1.3 \\
Myosalgia & 5 & 1.1 \\
\hline
\end{tabular}


lupus patients without $\mathrm{PH}$. They reported correlation of Raynaud's phenomenon with PH.

One hundred and seventy-eight patients $(38.2 \%)$ from 12 studies had arthritis, mostly proximal interphalangeal arthritis. Lupus is sometimes misdiagnosed as rheumatoid arthritis because arthritis may precede other system damages.

One hundred and twenty-nine patients $(27.7 \%)$ from eight studies had serous effusion-primarily pericardial effusion (88) and pleural effusion (10). In four studies, $57.3 \%$ (44.4-63.9 \%) of lupus patients with PH had serous effusion versus $21.7 \%(15.2-25 \%)$ of lupus patients without PH. They reported correlation of serous effusion with $\mathrm{PH}$.

One hundred and one patients $(21.7 \%)$ from six studies had serositis. In four studies, $60.5 \%(53.7-64.9 \%)$ of lupus patients with $\mathrm{PH}$ had serositis versus $29 \%$ (25.6-32.9 \%) of lupus patients without PH. They reported correlation of serositis with $\mathrm{PH}$.

TTE, electrocardiography, chest X-ray and right heart catheterization (RHC) were performed for the purpose of diagnosing $\mathrm{PH}$ in lupus patients. The pulmonary artery pressures of all 642 patients were measured by TTE. Two studies compared the sensitivity of TTE with chest X-ray and electrocardiography and reported the detection rates as 93.3, 26.7 and $26.7 \%$, respectively.

Among all the laboratory findings, positive ANA, positive ACL and positive RNP were significantly higher in the lupus patients with $\mathrm{PH}$. As seen in Table 3, the antiACL antibody in 198 patients (46.6\%) from 14 studies was positive. In five studies, $51.3 \%(46.2-56.8 \%)$ of lupus patients with $\mathrm{PH}$ had positive anti-ACL antibody versus $23.8 \%(16-28.9 \%)$ of lupus patients without $\mathrm{PH}$. They all reported positive correlation of the presence of anti-ACL antibody with the diagnosis of PH on TTE.

The anti-RNP antibody in 172 patients $(51.5 \%)$ from twelve studies was positive. In five studies, $49.2 \%$ (13-80.5\%) of lupus patients with PH had positive antiRNP antibody versus $20 \%(3.8-37.7 \%)$ of lupus patients without PH. They all reported positive correlation of the presence of anti-RNP antibody with the diagnosis of $\mathrm{PH}$ on TTE.

The anti- $\beta 2$-GPI antibody in 72 patients $(25 \%)$ from one study was positive; $25 \%$ of lupus patients with $\mathrm{PH}$ had positive anti- $\beta 2$-GPI antibody versus $12 \%$ of lupus patients without $\mathrm{PH}$. And it reported positive correlation of the presence of anti- $\beta 2$-GPI antibody with the diagnosis of PH on TTE.

Corticosteroids, immunosuppressants, vasodilators and anticoagulants were the commonly used medications for the patients in this analysis (Table 4).

All 273 patients $(42.5 \%$ ) from ten studies took two kinds of corticosteroids as basic treatment—oral administration
Table 3 Auto-antibodies of SLE patients with PAH

\begin{tabular}{lcr}
\hline Auto-antibodies & Patients & Positivity, $n(\%)$ \\
\hline ANA (+) & 230 & $222(96.5)$ \\
Ds-DNA (+) & 283 & $167(59.0)$ \\
RNP (+) & 334 & $172(51.5)$ \\
SSA (+) & 271 & $127(46.9)$ \\
ACL (+) & 425 & $198(46.6)$ \\
Rib (+) & 9 & $3(33.3)$ \\
Antithrombase (+) & 72 & $24(33.3)$ \\
SSB (+) & 245 & $73(29.8)$ \\
Antiplasmin (+) & 72 & $21(29.2)$ \\
Sm (+) & 257 & $68(26.5)$ \\
$\beta 2-G P I ~(+)$ & 72 & $18(25.0)$ \\
AHA (+) & 62 & $15(24.2)$ \\
ANCA (+) & 139 & $18(12.9)$ \\
Scl-70 (+) & 47 & $6(12.8)$ \\
Anti-APC (+) & 72 & $8(11.1)$ \\
Jo-1 (+) & 15 & $1(6.7)$ \\
T-PA (+) & 72 & $3(4.2)$ \\
\hline ANA & &
\end{tabular}

$A N A$ anti-nuclear antibody, Anti-ds-DNA anti-double-stranded DNA, $R N P$ anti-ribonucleoprotein antibody, SSA anti-Sjogren syndrome A antibody, $A C L$ anti-cardiolipin antibody, Rib anti-ribosome antibody, $S S B$ anti-Sjogren syndrome B antibody, Sm anti-Smith antigen, $\beta 2-G P I$ anti-cardiolipin- $\beta 2$-glycoprotein I complex, $A H A$ anti-histone antibody, ANCA anti-neutrophil cytoplasmic antibody, $\mathrm{Scl}-70$ anti-topoisomerase 1 antibody, $A P C$ anti-activated protein $\mathrm{C}$ antibody, $J o-1$ anti-histidyl-tRNA synthetase antibody, $T-P A$ anti-tissue-type plasminogen activator antibody

Table 4 Medicine treatment for 273 patients

\begin{tabular}{lcr}
\hline Medicines & Patients & $\%$ \\
\hline Corticosteroids & 268 & 98.2 \\
Immunosuppressant & 179 & 65.6 \\
Vasodilators & 143 & 52.4 \\
Anticoagulants & 9 & 3.3 \\
Other medicines & 23 & 8.4 \\
\hline
\end{tabular}

$(87.2 \%)$ and intravenous injection (11\%). Cyclophosphamide was used in 156 patients $(57.1 \%)$ from the same ten studies.

Two hundred and sixty-six patients $(41.4 \%)$ from nine studies were followed up for $0.75-12$ years (with a mean of 3.8 years). Forty-one of them (15.4\%) with PH died during the follow-up period. Only six studies reported the causes of death, which included right heart failure, kidney failure, severe infection, pulmonary embolism, cardiac arrest, arrhythmia, alimentary tract hemorrhage, cerebral vascular accident and sudden death. One hundred and thirteen patients $(42.5 \%)$ showed $23.8 \%(4.5-73.3 \%)$ 
improvement of their right ventricular systolic pressure by Doppler echocardiography after appropriate drug intervention. Sixty-eight patients $(25.6 \%)$ showed $11.6 \%$ $(1.8-61.7 \%)$ aggravation of $\mathrm{PH}$ after treatment.

\section{Discussion}

Pulmonary hypertension $(\mathrm{PH})$ has been reported in almost all rheumatic diseases including systemic sclerosis and SLE [14-17]. It is an increasingly recognized manifestation of SLE and an important reason for morbidity and mortality $[18,19]$. SLE with PH attracts more and more clinical and academic attentions.

It takes time for SLE-PH to be recognized and reported. In 1954, Harvey et al. [20] reported no PH in 138 SLE patients. In 1981, Perez and Kramer reported a $9 \%$ prevalence rate of PH in SLE patients [21]. The reported PH prevalence rates varied greatly depending on the type of study. Retrospective studies reported prevalence rates of 0.5-6\% [19], while prospective studies in asymptomatic patients found higher prevalence rates of 9-14\% [6, 22]. In this analysis, we found the prevalence rate of SLE-PAH in China ranged from 2.8 to $23.3 \%$ as reported by twelve retrospective studies. The wide variability in the reported prevalence rates reflects the varying definitions of $\mathrm{PH}$, differences in diagnostic methods, population groups studied and number of patients involved [23]. The prevalence rate from prospective cross-sectional cohort study would be better than the retrospective smaller studies, but no reports were found yet.

It is noteworthy that more than half of patients in this analysis originated from the three big metropolitan cities (Beijing, Shanghai and Guangzhou) in China. This may not necessarily indicate that the prevalence of SLE-PH in the three places was higher than the rest of China. It may simply be that these cities have more medical resources and expertise in recognizing and diagnosing $\mathrm{PH}$ and patients there can better afford the costs of medical care.

The pathogenesis of PH in SLE is not well understood. Abnormal vasospasm, Raynaud's phenomenon, platelet abnormalities, vasculitis, thrombosis and thromboembolism have been suggested as possible pathogenetic factors $[8,24,25]$. Endothelial dysfunction is strongly implicated in the pathogenesis of autoimmune vasculitic diseases including lupus [26, 27]. Nakamura et al. [28] found the elevation of endothelin may be associated with $\mathrm{PH}$ and possibly related to disease activity in a murine lupus model. Shen et al. [29] reported that PH patients had higher serum endothelin more commonly in the active stage and the level of endothelin was correlated with pulmonary pressure. In addition, a variety of auto-antibodies in vivo of lupus patients can cause vascular endothelial cell injury such as ACL, LA, ANA, ds-DNA. They could directly damage the endothelial cells or form immune complexes and then deposit in the vascular wall, leading to vasoconstriction, platelet aggregation and the formation of thrombosis. In our study, we found that positive ACL and positive RNP were correlated with $\mathrm{PH}$ and they were strong predictors of $\mathrm{PH}$ in some studies, which are compatible with previous findings [30-32]. Only one study reported $25 \%$ anti- $\beta 2$-GPI antibody was positive in 72 SLE-PH patients, which is consistent with Houman's research studied in Tunisia ( $25 \%$ of positive for anti$\beta 2$-GPI in 100 patients) [33].

Most cases of SLE-associated PH are mild, which makes early diagnosis difficult, and only those cases presenting with severe PH have been reported. Lian et al. [30] suggested that more than $40 \%$ of the SLE-PH patients would not have observable symptoms when early $\mathrm{PH}$ persisted. In general, the clinical symptoms of SLE patients with PH are nonspecific such as progressively exertional dyspnea, chest pain, nonproductive cough, edema, easy fatigue, impaired exercise tolerance [14], and can also be caused by many other factors including pleural or pericardial effusions, interstitial lung disease, making it possible to miss the diagnosis [34]. Physical findings include a loud second pulmonic heart sound, pedal edema, serous effusion, Raynaud's phenomenon and so on. In this study, the nonspecific symptoms reported above were not so common as Raynaud's phenomenon and serous effusion. One hundred and ninety-three patients $(41.4 \%)$ presented Raynaud's phenomenon. Raynaud's phenomenon is characterized by vasospasm and could be the cutaneous manifestation of general vascular disorder resulting in pulmonary arterial vasoconstriction [3,35]. The high frequency of Raynaud's phenomenon patients with SLE and PH suggests a potential role for etiology of $\mathrm{PH}$, and also supports the notion that Raynaud's phenomenon is a pathogenic risk factor and a marker for disease severity [30, 36].

TTE has been widely used to screen for PH due to its safety, convenience and sensitivity. Denton et al. [37] reported that TTE had a sensitivity of $90 \%$ and a specificity of $75 \%$ compared with RHC. Gonzalez et al. [38] compared TTE with RHC to detect PH and found that the method of applying differential pressure of tricuspid regurgitation for estimating pulmonary hypertension had high accuracy with a sensitivity of $83.3-90 \%$ and a specificity of $75 \%$. RHC is considered the gold standard test in the diagnosis and classification of $\mathrm{PH}$. PH may be caused by venous or arterial changes, and pulmonary vasculitis in SLE patients can lead to pulmonary arterial hypertension (PAH). RHC is currently the only way to differentiate what kind of PH that SLE patients suffered from. Besides, with an assessment of vasoreactivity, RHC is necessary if $\mathrm{PH}$ is highly suspected and is needed for 
decision making with particular respect to treatment. Jais et al. [39] emphasized that RHC was mandatory before starting any treatment and for following-up PAH patients and showed it provided strong predictors of response to immunosuppressive therapy by reliably measuring cardiac index and calculating pulmonary vascular resistance.

Insidious onset of SLE-associated PH develops rapidly. It is irreversible and usually of poor outcome with a 2- to 5-year survival after initial diagnosis [40]. Therefore, early, intensive treatment for the primary disease (SLE) is the key to improve prognosis. Various drugs have been used for the treatment of SLE-associated PH, such as corticosteroids, immunosuppressants, vasodilators, endothelin receptor antagonists and anticoagulants. They could improve the symptoms of lupus, and only some of them had an effect on decreasing the pulmonary artery pressure. But there was a lack of detailed comparison of the results of different combined therapies. Corticosteroids plus immunosuppressants can block the immune-mediated vascular inflammation in the early stage. Promising results have been obtained with combination therapy that includes steroids and cyclophosphamide in patients with early stages of $\mathrm{PH}$ [22]. Abnormal pulmonary and cardiac function can be improved by immunosuppressants and corticosteroids alone or combined with pulmonary vasodilators [39]. If a patient is strongly vasodilator responsive, then treatment with high dosage of calcium channel blocker should be adequate [34]. It has been proved that traditional vasodilators could improve hemodynamics, increase exercise tolerance and prolong survival time by reducing right ventricular afterload. However, there are potential undesirable response such as an increase in ventilation/perfusion mismatch and a reduction in systemic vascular resistance, leading to hypotension [20, 41]. If a patient is not vasodilator responsive, then treatment would include endothelin receptor blockers [34]. There are two kinds of endothelin receptor blockers: selective and nonselective. Bosentan, a nonselective endothelin receptor blocker, was reported by Ahmadi-Simab et al. [42] to have a good effect on relieving PH. Mok et al. [43] found 12 months of bosentan treatment resulted in significant improvement in sixminute walk distance (6MWD), transient or sustained drop in systolic PAP. Because of endothelial dysfunction in lupus patients with $\mathrm{PH}$, long-term hypoxia and hypercapnia lead to secondary polycythemia, hypercoagulability, stasis of the microcirculation, and then increasing risk of thrombosis and embolism. Thus, anticoagulant therapy has been recommended to be used with other medicines such as corticosteroids and immunosuppressants. However, the effect of warfarin anticoagulation is controversial for the treatment of idiopathic PAH patients, and there is no evidence to support that anticoagulant therapy provides benefit for SLE-PAH patients.
Some Chinese herbals may also relieve PH. Tang et al. [44] found ligustrazine extracted from ligustici could decrease the level of endothelin through inhibiting the expression and release of ET-1 in hypoxic PH rats. Jiang et al. [45] observed salvia miltiorrhiza may depress the secretion of endothelin and then reduce hypoxic pulmonary vasoconstriction. Gene therapies for $\mathrm{PH}$ have been successful in the laboratory with the potential for effective treatments. Lung transplantation is the only measure to treat advanced $\mathrm{PH}$, and 1-year survival rate after transplantation is $65-70 \%$.

In general, the prognosis in mild and moderate disease is not certain, depends on different therapies and courses of treatment. However, SLE patients with severe symptomatic PH carry a poor prognosis. Oren et al. [46] reported the overall 2-year survival was $\leq 50 \%$ when pulmonary hypertension was present. Chung et al. [47] reported PH in SLE untreated had a 3-year mortality of $54.1 \%$. In recent years, SLE patients have improved survival rate closer to idiopathic PAH with the advent of PAH-specific therapies [48, 49].

A major limitation of this study is that RHC confirmation of $\mathrm{PH}$ was not performed on all patients. PASP $>30 \mathrm{mmHg}$ on TTE in lupus patients was chosen a priori, and there are concerns about TTE's sensitivity and specificity. In addition, most studies were retrospective with small sample sizes. Therefore, multi-center prospective cohort studies of SLE patients with $\mathrm{PH}$ are called for in China.

In conclusion, $\mathrm{PH}$ is an increasingly recognized complication in SLE. PH associated with SLE typically affects middle-age women and is often characterized by nonspecific symptoms and poor prognosis. Early diagnosis is essential for SLE patients with PH. Gold standard RHC is strongly recommended since symptoms of $\mathrm{PH}$ in lupus patients are nonspecific, especially for those who had PASP $>30 \mathrm{mmHg}$ on TTE with positive anti-ACL or antiRNP antibodies or Raynaud's phenomenon or serous effusion or serositis. Early identification, standard $\mathrm{PH}$ treatment as well as intensive treatment for SLE can improve the prognosis.

Open Access This article is distributed under the terms of the Creative Commons Attribution License which permits any use, distribution, and reproduction in any medium, provided the original author(s) and the source are credited.

\section{References}

1. Sanchez O, Sitbon O, Garcia G et al (2003) Pulmonary artery hypertension associated with connective tissue diseases. Presse Med 32:789-799 
2. Chen LJ, Chang HC, Lu LY et al (2004) Prolonged survival after single lung transplantation for pulmonary hypertension secondary to systemic lupus erythematosus. J Chin Med Assoc 67:248-251

3. Haas C (2004) Pulmonary hypertension associated with systemic lupus erythematosus. Bull Acad Natl Med 188:985-997 discussion 997

4. Yoshio T, Masuyama J, Sumiva M et al (1994) Antiendothelial cell antibodies and their relation to pulmonary hypertension in systemic lupus erythematosus. J Rheumatol 11:2058-2063

5. Hassoun PM (2009) Pulmonary arterial hypertension complicating connective tissue diseases. Semin Respir Crit Care Med 30:429-439. doi:10.1055/s-0029-1233312

6. Winslow TM, Ossipov MA, Fazio GP et al (1995) Five-year follow-up study of the prevalence and progression of pulmonary hypertension in systemic lupus erythematosus. Am Heart J 129: 510-515

7. Turner A, Samanta A, Nichol FE (1991) Primary pulmonary hypertension associated with systemic lupus erythematosus. Clin Rheumatol 10:320-322

8. Asherson RA, Higenbottam TW, Dinh Xuan AT et al (1990) Pulmonary hypertension in a lupus clinic: experience with twenty-four patients. J Rheumatol 17:1292-1298

9. Quismorio FP Jr, Sharma O et al (1984) Immunopathologic and clinical studies in pulmonary hypertension associated with systemic lupus erythematosus. Semin Arthritis Rheum 13:349-359

10. Kim WU, Min JK, Lee SH et al (1999) Causes of death in Korean patients with systemic lupus erythematosus: a single center retrospective study. Clin Exp Rheumatol 17:539-545

11. Luo RQ, Lei YX, Zhang X et al (2008) Clinical analysis of patients with systemic lupus erythematosus and concomitant pulmonary hypertension. Nan Fang Yi Ke Da Xue Xue Bao 28:1860-1863

12. Li EK, Tam LS (1999) Pulmonary hypertension in systemic lupus erythematosus: clinical association and survival in 18 patients. J Rheumatol 26:1923-1929

13. Tan EM, Cohen AS, Fries JF et al (1982) The 1982 revised criteria for the classification of systemic lupus erythematosus. Arthritis Rheum 11:1271-1277

14. Hasegawa EM, Caleiro MT, Fuller R et al (2009) The frequency of anti-beta2-glycoprotein I antibodies is low and these antibodies are associated with pulmonary hypertension in mixed connective tissue disease. Lupus 18:618-621. doi:10.1177/0961203308101719

15. Frea S, Capriolo M, Marra WG et al (2011) Echo Doppler predictors of pulmonary artery hypertension in patients with systemic sclerosis. Echocardiography 28:860-869. doi:10.1111/j.15408175.2011.01467.x

16. Shariff N, Kumar A, Narang R et al (2007) A study of pulmonary arterial hypertension in patients with rheumatoid arthritis. Int $\mathbf{J}$ Cardiol 115:75-76. doi:10.1016/j.ijcard.2006.01.013

17. Arca Barca B, Paredes Vila S, Mazaira Riocabo A (2011) Primary pulmonary hypertension in Sjogren's syndrome: a rare association. Reumatol Clin 7:212. doi:10.1016/j.reuma.2010. 06.006

18. Cefle A, Inanc M, Sayarlioglu M et al (2011) Pulmonary hypertension in systemic lupus erythematosus: relationship with antiphospholipid antibodies and severe disease outcome. Rheumatol Int 31:183-189. doi:10.1007/s00296-009-1255-2

19. Magliano M, Isenberg DA, Hillson J (2002) Pulmonary hypertension in autoimmune rheumatic diseases: where are we now? Arthritis Rheum 46:1997-2009. doi:10.1002/art.10442

20. Harvey AM, Shulman LE, Tumulty PA et al (1954) Systemic lupus erythematosus: review of the literature and clinical analysis of 138 cases. Medicine (Baltimore) 33:291-437

21. Perez HD, Kramer N (1981) Pulmonary hypertension in systemic lupus erythematosus: report of four cases and review of the literature.
Semin Arthritis Rheum 11:177-181. doi:10.1016/0049-0172(81) 90098-6

22. Tanaka E, Harigai M, Tanaka M et al (2002) Pulmonary hypertension in systemic lupus erythematosus: evaluation of clinical characteristics and response to immunosuppressive treatment. J Rheumatol 29:282-287

23. Prabu A, Patel K, Yee CS et al (2009) Prevalence and risk factors for pulmonary arterial hypertension in patients with lupus. Rheumatology (Oxford) 48:1506-1511. doi:10.1093/rheumatology/ kep203

24. Koyama S, Ichiyoshi T, Chino M et al (1996) Systemic lupus erythematosus with pulmonary hypertension. Intern Med 35: 39-42

25. Farber HW, Loscalzo J (2004) Pulmonary arterial hypertension. N Engl J Med 351:1655-1665. doi:10.1056/NEJMra035488

26. Pearson JD (2000) Normal endothelial cell function. Lupus 9:183188

27. Humbert M, Simonneau G (2005) Drug Insight: endothelinreceptor antagonists for pulmonary arterial hypertension in systemic rheumatic diseases. Nat Clin Pract Rheumatol 1:93-101. doi:10.1038/ncprheum0048

28. Nakamura T, Ebihara I, Tomino Y et al (1995) Effect of a specific endothelin A receptor antagonist on murine lupus nephritis. Kidney Int 47:481-489

29. Shen JY, Chen SL, Wu YX et al (1999) Pulmonary hypertension in systemic lupus erythematosus. Rheumatol Int 18:147-151

30. Lian F, Chen D, Wang Y et al (2011) Clinical features and independent predictors of pulmonary arterial hypertension in systemic lupus erythematosus. Rheumatol Int. doi: 10.1007/ s00296-011-1880-4

31. Grunig E, Ehlken N, Nagel C (2008) Anticoagulation in pulmonary arterial hypertension. Hamostaseologie 28:225-230. doi: 08040225[pii]

32. Cefle A, Inanc M, Sayarlioglu M et al (2011) Pulmonary hypertension in systemic lupus erythematosus: relationship with antiphospholipid antibodies and severe disease outcome. Rheumatol Int 31:183-189. doi:10.1007/s00296-009-1255-2

33. Houman MH, Smiti-Khanfir M, Ben Ghorbell I et al (2004) Systemic lupus erythematosus in Tunisia: demographic and clinical analysis of 100 patients. Lupus 13:204-211

34. Pope $\mathbf{J}$ (2008) An update in pulmonary hypertension in systemic lupus erythematosus: do we need to know about it? Lupus 17:274-277

35. Levien TL (2010) Advances in the treatment of Raynaud's phenomenon. Vasc Health Risk Manag 6:167-177

36. Kasparian A, Floros A, Gialafos E et al (2007) Raynaud's phenomenon is correlated with elevated systolic pulmonary arterial pressure in patients with systemic lupus erythematosus. Lupus 16:505-508. doi:10.1177/0961203307080629

37. Denton CP, Cailes JB, Phillips GD et al (1997) Comparison of Doppler echocardiography and right heart catheterization to assess pulmonary hypertension in systemic sclerosis. $\mathrm{Br} \mathrm{J}$ Rheumatol 36:239-243

38. Gonzalez-Lopez L, Cardona-Munoz EG, Celis A et al (2004) Therapy with intermittent pulse cyclophosphamide for pulmonary hypertension associated with systemic lupus erythematosus. Lupus 13:105-112

39. Jais X, Launay D, Yaici A et al (2008) Immunosuppressive therapy in lupus- and mixed connective tissue disease-associated pulmonary arterial hypertension: a retrospective analysis of twenty-three cases. Arthritis Rheum 58:521-531. doi:10.1002/ art.23303

40. Pan TL, Thumboo J, Boey ML (2000) Primary and secondary pulmonary hypertension in systemic lupus erythematosus. Lupus 9:338-342 
41. Robbins IM, Christman BW, Newman JH et al (1998) A survey of diagnostic practices and the use of epoprostenol in patients with primary pulmonary hypertension. Chest 114:1269-1275

42. Ahmadi-Simab K, Lamprecht P, Gross WL (2005) Successful therapy of bosentan-refractory pulmonary arterial hypertension (PH) with inhalative iloprost. Clin Exp Rheumatol 23:402-403

43. Mok MY, Tsang PL, Lam YM et al (2007) Bosentan use in systemic lupus erythematosus patients with pulmonary arterial hypertension. Lupus 16:279-285. doi:10.1177/0961203307076509

44. Tang TQ, Huang NS, Chen SF et al (1999) The experimental study of the effects of ligustrazine on the blood serum endothelin and on the expression of ET-1 of the lung and ETA-RmRNA of the pulmonary vascular smooth muscle cells in hypoxic rats. Chin Pharm J 34:17-20

45. Jiang Z, Liu HX, Yuan JS et al (1997) Effects of hypoxia and salvia miltiorrhiza on the secretion of endothelin and releasing of nitrogen monoxidum in pulmonary artery endothelial cell. Chin J Intern Med 36:778-779

46. Orens JB, Martinez FJ, Lynch JP (1994) Pleuropulmonary manifestations of systemic lupus erythematosus. Rheum Dis Clin North Am 20:159-193

47. Chung SM, Lee CK, Lee EY et al (2006) Clinical aspects of pulmonary hypertension in patients with systemic lupus erythematosus and in patients with idiopathic pulmonary arterial hypertension. Clin Rheumatol 25:866-872. doi:10.1007/s10067-006-0206-5

48. Chung L, Liu J, Parsons L et al (2010) Characterization of connective tissue disease-associated pulmonary arterial hypertension from REVEAL: identifying systemic sclerosis as a unique phenotype. Chest 138:1383-1394. doi:10.1378/chest.10-0260

49. Zhang R, Dai LZ, Xie WP et al (2011) Survival of Chinese patients with pulmonary arterial hypertension in the modern treatment era. Chest 140:301-309. doi:10.1378/chest.10-2327 